\section{A TECHNIQUE TO SIMPLIFY AND IMPROVE EXPOSURE IN HEART-LUNG TRANSPLANTATION}

Heart-lung transplantation is associated with high perioperative mortality rates. A modified operative technique was used by one surgeon operating on 17 patients at the University of Arizona, Tucson, and the Inland Northwest Thoracic Organ Transplant Program, Spokane, Washington. This technique gives greater exposure to the area of dissection behind the heart-lung block after implantation and makes maintaining hemostasis easier. No deaths from bleeding complications occurred and no reoperations for bleeding were required with this technique. The Kaplan-Meier survival was $82 \%$ at 1 year. This technique simplifies a difficult technical procedure and may reduce mortality rate. (J THORAC CARDIOVASC SURG 1995;110:1590-3)

Timothy B. Icenogle, MD, ${ }^{\mathrm{a}, \mathrm{b}}$ and Jack G. Copeland, MD, ${ }^{\mathrm{c}}$ Spokane, Wash., and Tucson, Ariz.
$\mathrm{H}$ eart-lung transplantation is the procedure of choice for the treatment of selected conditions requiring replacement of both heart and lungs. It is also an alternative for replacement of the lungs when the heart can be used in a domino operation. The 1-year survival for heart-lung transplantation is $59 \%$, and about one third of all deaths are the result of intraoperative technical complications such as hemorrhage and primary graft failure. ${ }^{1}$

The heart-lung surgical technique was modified to simplify the procedure and to improve exposure behind the heart-lung block, often a site of troublesome bleeding. Improved exposure behind the heart-lung block may reduce morbidity and mortality.

\section{Methods}

During a 51/2-year period between January 1988 and May 1993, seventeen patients underwent heart-lung transplantation by one surgeon (T.B.I.). Four operations were performed at the University of Arizona (Tucson) and 13 were performed at Sacred Heart Medical Center (Spokane, Wash.). Five operations were domino procedures in

From Inland Northwest Thoracic Organ Transplant Program, Sacred Heart Medical Center, Heart Institute of Spokane, ${ }^{a}$ Health Research Education Center, Washington State University, ${ }^{\mathrm{b}}$ Spokane, and the Section of Cardiothoracic Surgery, The University of Arizona, Tucson. ${ }^{c}$

Received for publication April 4, 1994.

Accepted for publication April 6, 1995.

Address for reprints: Timothy B. Icenogle, MD, N.W. Cardiothoracic/Transplant Surgeons, Suite 532 (Center Tower), Sacred Heart Doctors Bldg., 105 W. Eighth Ave., Spokane, WA 99204-2236.

Copyright $(1995$ by Mosby-Year Book, Inc.

$0022-5223 / 95 \$ 5.00+0 \quad \mathbf{1 2 / 1 / 6 5 3 3 6}$ which the heart-lung recipient's native heart was transplanted into another patient.

Recipient operative technique. A median sternotomy is made, the pleura is entered on both sides, and the edges of the pericardium are held with three sutures for manipulation. The venae cavae are cannulated through the atrium, caval tapes are placed around each vena cava, and the aorta is cannulated.

Once cardiopulmonary bypass has begun, the caval tapes are secured, the aorta is crossclamped and the heart is resected as in heart transplantation. The left atrium is divided midway between the right and left pulmonary veins and then the veins are stapled with disposable TA 30 Long-V3 surgical staplers (AutoSuture; United States Surgical Corp., Norwalk Conn.; Fig. 1). These staplers have a smooth contour for easy passage around structures, are not reloaded, and allow rapid use. The left pulmonary veins are divided proximal to the staple line and discarded. The right pulmonary veins and attached left atrial remnant are separated from the right atrium at Waterson's groove. The pulmonary artery is dissected; the right branch is stapled with vascular staples at its junction with the pericardium and the left is dissected cautiously to protect the recurrent laryngeal nerve and then stapled. The left lung is brought into view and the inferior pulmonary ligament is divided with electrocautery. A Proximate TLH-90 Heavy Wire stapler (Ethicon, Inc., Somerville, N.J.) is used to staple the hilus of the lung, then the lung is removed with electrocautery distal to the stapling instrument. This particular stapler is less susceptible to twisting of the jaws and is ideal for the hilus. These steps are repeated on the right side.

The right pericardium is incised along the pericardiodiaphragmatic junction down to approximately $2 \mathrm{~cm}$ from the phrenic nerve. The dissection is carried superiorly running parallel to the phrenic nerve to create a pericardial fat-pad flap with which to wrap the tracheal anastomosis (Fig. 2). The left pleural membrane is divided one third the distance from the diaphragm to the aorta in an anterior-to-posterior direction, with the dissection ending $2 \mathrm{~cm}$ away from the phrenic nerve. This creates a T-shaped 


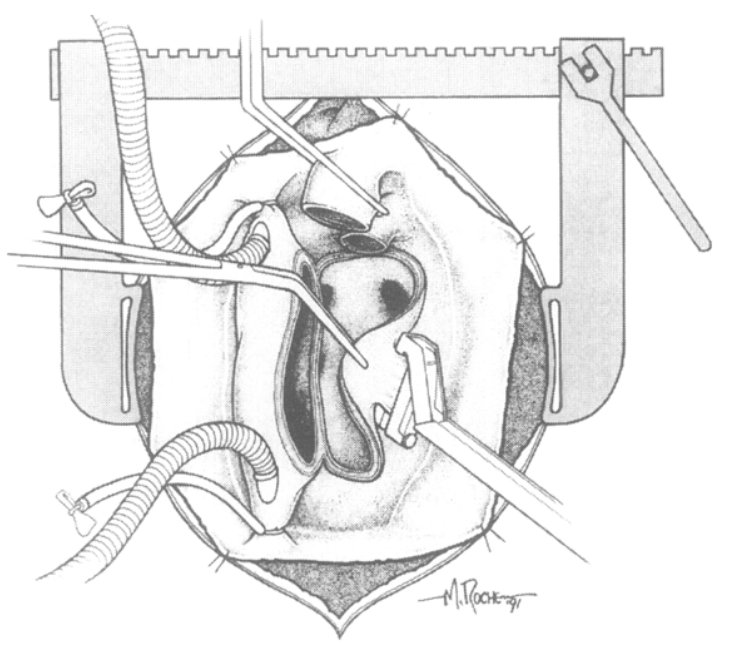

Fig. 1. Stapling of the left inferior pulmonary vein after resection of the heart.

cut in the left pericardium. Stay sutures keep tension on the lower flap to support the heart during implantation.

An inverted $\mathrm{Y}$ incision is made over the carina and mainstem bronchi in the posterior pericardium. The bronchi are entered with the cautery just below the carina. Traction is placed on each bronchus with a clamp and the bronchi are dissected to the hilar staple line with the cautery. The vagal nerves are identified and protected during this dissection. It is imperative to obtain hemostasis in the mediastinum at this point because this is the most likely site of troublesome bleeding once bypass is discontinued. The Argon Beam Coagulator (C. R. Bard Inc., Murray Hill, N.J.) is effective in controlling small vessels without causing deep-tissue necrosis. It is used liberally in the recipient operative technique.

The heart-lung block is brought onto the field and the end of an intravenous tube is placed into the amputated left atrial appendage and secured. Saline solution at $4^{\circ} \mathrm{C}$ is infused into the left atrium to cool the heart and evacuate air. The Argon Beam Coagulator is used to cauterize the posterior aspect of the heart-lung block. The stapled end of the trachea is amputated one ring above the carina.

The heart-lung block is placed in the chest, and the right lung is passed posterior to the right atrium and into the right chest through the pericardial incision anterior to the phrenic nerve (Fig. 3). The left lung falls into the left chest anterior to the pericardium and the phrenic nerve. A stay suture is placed with tension on the left inferior pericardial flap to support the heart and reduce tension transferred to the trachea during tracheal anastomosis. The tracheal anastomosis is sutured with a single 3-0 Prolene suture (Ethicon) run in a simple over-and-over fashion. The right atrium is anastomosed with a 3-0 Prolene suture and the aorta is anastomosed with a 4-0 Prolene suture. The patient is placed in a steep Trendelenberg's position, a split angiocatheter is placed into the aorta, and the crossclamp is removed. Air is also removed from the left ventricle with a split angiocatheter.

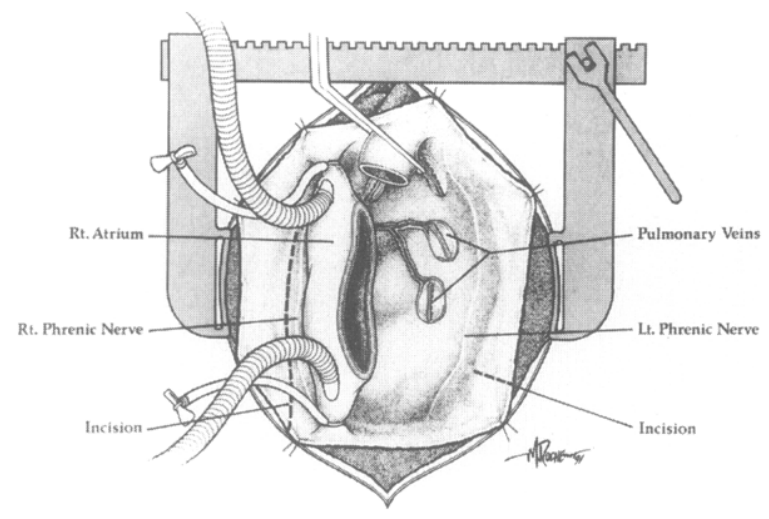

Fig. 2. Exposure after resection of the left atrium. Lines of incision in the pericardium are shown by the dashed line.

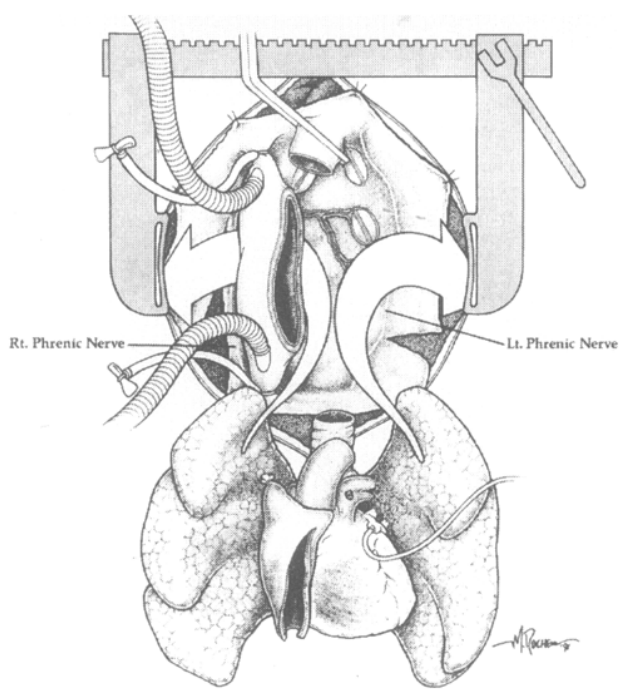

Fig. 3. The heart-lung block is placed anterior to the phrenic nerves. (Redrawn with permission from MosbyYear Book, Inc., Chicago, Ill. Original drawing taken from Donald B. Doty's Cardiac Surgery: A Looseleaf Workbook and Update Service. Chicago: Mosby-Year Book, 1989: Special Problems, page TRANSPL 19.)

An intensive effort is made to isolate areas of bleeding before weaning from bypass. Frequently, diffuse oozing prevents isolation of bleeding sites until after the protamine is given. Placement of the lungs anterior to the phrenic nerves offers the ability to check behind the heart-lung block for brief periods to find areas of bleeding. The surgeon may place a hand behind the apex of the left lung and pull the entire lung from the hemithorax to view the bronchial dissection site on the left side (Fig. 4). This maneuver is surprisingly well tolerated for brief periods. The same maneuver can be done on the right side (Fig. 5). The entire posterior mediastinal dissection can be 


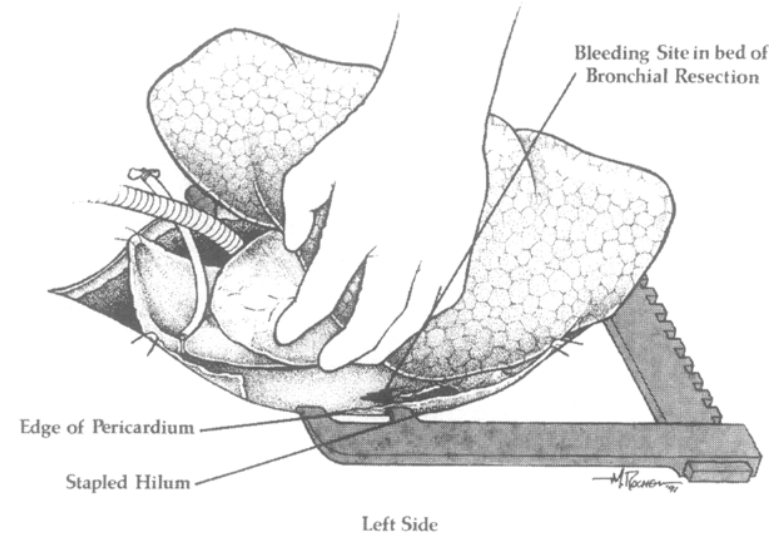

Fig. 4. Exposure of the area posterior to the heart-lung block from the left side.

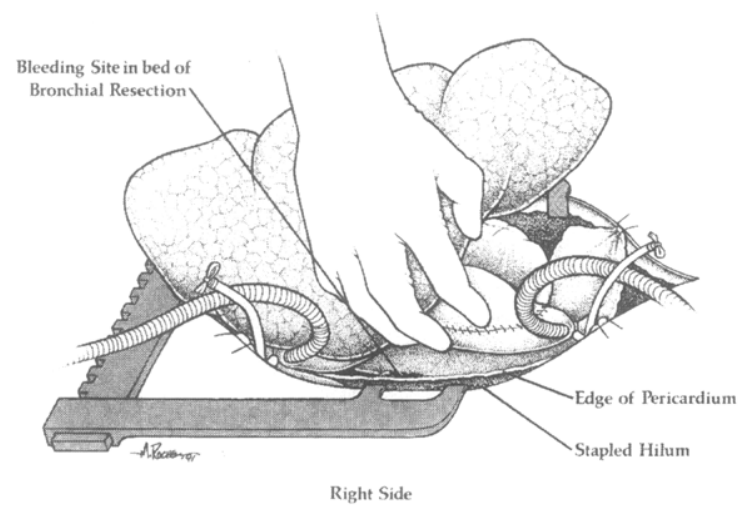

Fig. 5. Exposure of the area posterior to the heart-lung block from the right side.

brought into view with this technique (Fig. 6). Visibility of the bleeding sites on the posterior mediastinum enables the surgeon to control these sites and thus reduces the incidence of postoperative bleeding.

Statistical analysis. Survival data were analyzed with the Kaplan-Meier actuarial survival method.

\section{Results}

Seventeen heart-lung transplant operations were performed with this technique. Five were domino procedures. Indications for transplantation were primary pulmonary hypertension (nine), Eisenmenger's syndrome (two), $\alpha_{1}$-antitrypsin deficiency (three), chronic obstructive pulmonary disease (two), and bronchiectasis (one).

Four patients had undergone previous thoracic operations. Three patients had previous repair of atrial septal defects, and one patient had a previous thoracotomy for a Blalock-Taussig shunt as an in-

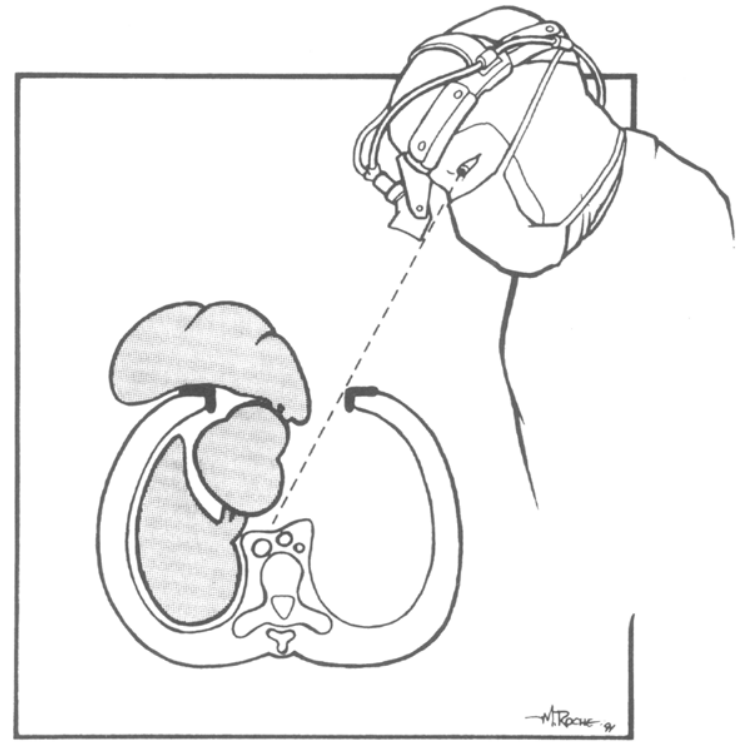

Fig. 6. Rolling the heart-lung block gives exposure to the area of bronchial dissection.

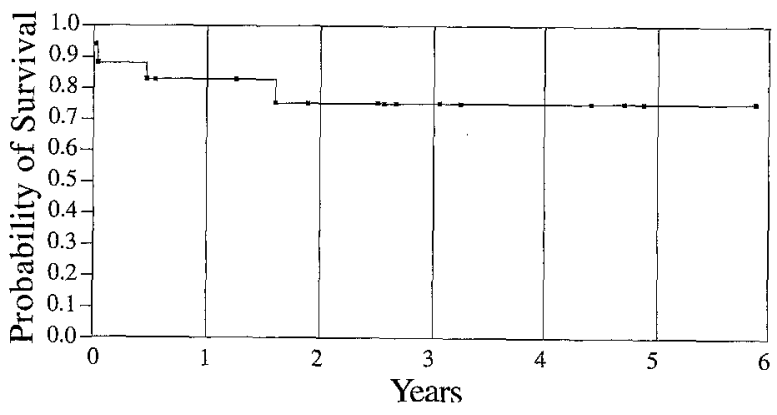

Fig. 7. Survival curve for heart-lung transplantation.

fant and a median sternotomy for an aortic valve replacement at the age of 10 years.

Mean follow-up time was 878 days. No patient died of hemorrhage at operation or of bleeding complications later, and no patient required reoperation for bleeding. The 1-year Kaplan-Meier actuarial survival was $82 \%$, and the 2- and 3-year survivals were each $75 \%$ in this series (Fig. 7). There were no problems with tracheal healing. There were no instances of phrenic nerve palsy or recurrent laryngeal nerve palsy. One patient had problems with a bezoar, presumably as a result of vagal nerve injury.

There were four deaths in this series. One perioperative death was that of a critically ill 21 -year-old man with primary pulmonary hypertension and decompensated failure of the right side of the heart. 
He died of unrecognized septicemia shortly after transplantation. After operation, screening blood cultures drawn before transplantation showed gramnegative and gram-positive bacteria, which were later localized to a central line site.

The second perioperative death was that of a patient who had an embolism from a thrombosed aortic valve, which was caused by a lupus-associated hypercoagulable state 3 weeks after operation. Another patient died of Aspergillus pneumonia 6 months after operation. A fourth patient died of bronchiolitis obliterans at 20 months after operation.

Three patients in this series had noncompliant lungs and high inspiratory pressures immediately after implantation. They had hemodynamic instability on attempts to retract the lung, and the maneuver to expose the bronchial dissection area was therefore abandoned. Fortunately, hemostasis was achieved in two patients despite inability to check this area. The patient with unknown line septicemia died shortly after operation, so the presence or absence of hemostasis could not be determined.

\section{Discussion}

Few centers in the United States perform heartlung transplantation with regularity, and the ability to learn the operation is limited. The technical difficulty of the heart-lung transplant operation and the demanding early postoperative period are reflected in a high operative (less than 30-day) mortality rate. It is somber testimony to the importance of these factors that, after 10 years of experience, $16 \%$ to $20 \%$ of patients still die during the perioperative period. $^{1,2}$ The operative mortality rate contributes significantly to the relatively low 1-year survival of only $59 \% .{ }^{1,2}$ The high operative mortality rates and low 1-year survival rates are a deterrent for transplant programs that otherwise might consider offering this procedure.

The main causes of operative death are primary graft failure, bleeding, and tracheal dehiscence. Primary graft failure may be avoided by better preservation techniques and donor selection. Changes in operative technique may reduce deaths caused by hemorrhage and problems with tracheal healing. Several authors have described modifications of the technique originally described by Jamieson and coworkers ${ }^{3}$ at Stanford. Haverich and colleagues ${ }^{4}$ suggested alternatives to the use of the omentum to wrap the tracheal anastomosis and protect it from dehiscence. Modifications in technique intended specifically to reduce bleeding have been described and may contribute to a reduced mortality rate. ${ }^{5,6}$

The technique presented here is relatively simple and allows the surgeon to view the area behind the heart-lung block. The tracheal and bronchial dissection areas are frequently a source of bleeding and are nearly impossible to see if the heart-lung block is tethered behind the phrenic nerves. The ability to see this well-vascularized area and obtain hemostasis may decrease blood loss and lower the operative mortality rate.

There were no adverse sequelae in this series from placement of the lungs anterior to the phrenic nerve. Initial concern regarding the possibility of pulmonary venous compression was not justified. The heart tended to fall into the left side of the chest during the implantation procedure unless it was supported by the pericardial flap. Once the chest was closed, the heart was supported by the inflated lungs and assumed its usual position.

This technique simplifies the operative procedure and provides the ability to control hemorrhage behind the heart-lung block. Avoidance of frustrating hemorrhagic complications in these desperately ill patients may improve 1-year survival. We believe that this approach may increase the willingness of some centers to offer heart-lung transplantation as part of their clinical transplantation services.

\section{REFERENCES}

1. Kriett JM, Kaye MP. The registry of the International Society for Heart and Lung Transplantation: eighth official report-1991. J Heart Lung Transplant 1991; 10:491-8.

2. Kaye MP. The registry of the International Society for Heart and Lung transplantation: ninth official report-1992. J Heart Lung Transplant 1992;11:599606.

3. Jamieson SW, Stinson EB, Oyer PE, et al. Operative technique for heart-lung transplantation. J THORAC CARdiovasc SuRg 1984;87:930-5.

4. Haverich A, Frimpong-Boateng K, Wahlers T, Schäfers H-J. Pericardial flap-plasty for protection of the tracheal anastomosis in heart-lung transplantation. $J$ Cardiac Surg 1989;4:136-9.

5. Vouhé PR, Dartevell PG. Heart-lung transplantation: technical modifications that may improve the early outcome. J THORAC CARdiovasc Surg 1989;97: 906-10.

6. Novick RJ, Menkis AH, McKenzie FN, et al. Reduction in bleeding after heart-lung transplantation: the importance of posterior mediastinal hemostasis. Chest 1990;98:1383-7. 